\title{
Active Search for Epileptiform Electroencephalogram Activity by External Stimulation in Critically Ill Patients
}

\author{
Fábio A. Nascimento, Felippe Borlot, Danah Aljaafari, Martin del Campo
}

Keywords: Epilepsy, EEG, SIRPIDs, Neurocritical Care, Critical Care, Electroencephalography

A 68-year-old woman presented with a 2-day history of fluctuating level of consciousness. She underwent an electroencephalogram (EEG) to rule out nonconvulsive status epilepticus. Nail bed pressure applied to the extremities triggered paroxysms of brain activity consistent with stimulus-induced rhythmic, periodic, or ictal discharges (SIRPIDs) (Fig. 1) not accompanied by clinical manifestations. The SIRPIDs pattern consisted of 10- to 15-second runs of paroxysmal, medium- to high-amplitude polymorphic 1 to $2 \mathrm{~Hz}$ delta (mixed with $4-5 \mathrm{~Hz}$ theta), alternating with nonrhythmic high-amplitude waveforms, appearing diffusely throughout the brain (but frontally accentuated). At certain times, it was triphasically configured; at other times, it was clearly epileptiform. Magnetic resonance imaging scan of the brain showed multiple areas of diffuse restriction of varying ages involving the right periventricular white matter (Fig. 2A) and extending into the posterior right lentiform nucleus (Fig. 2B) as well as encephalomalacia in the left centrum semiovale (Fig. 2C). These radiological abnormalities were considered secondary to primary central nervous system vasculitis to which she succumbed. The SIRPIDs observed in this patient were likely a sign of cortical irritability; nonetheless, there was not strong evidence suggesting that it contributed to her demise.

SIRPIDs were first described by Hirsch et al as periodic, rhythmic, or ictal-appearing discharges consistently induced by alerting stimuli. ${ }^{1}$ Although being underrecognized and underreported, SIRPIDs seem to be relatively common. According to the original report, this EEG pattern may be found in roughly one-fifth of critically ill patients undergoing continuous EEG monitoring. ${ }^{1}$ This EEG phenomenon is believed to be epileptogenic when associated with clinical manifestations that are stereotyped and temporally linked with the discharges. Etiologically, SIRPIDs are associated with a wide variety of conditions, including intracranial hemorrhages, cerebral infarctions, hypoxic-ischemic brain injury, traumatic brain injury, and neurodegenerative diseases such as Creutzfeldt-Jakob disease. ${ }^{1-4}$ In terms of pathophysiology, the mechanisms underlying
SIRPIDs are still unknown. ${ }^{1,5}$ Similarly, their overall prognostic significance is not fully understood. ${ }^{1,6}$ However, in comatose survivors of cardiac arrest who underwent therapeutic hypothermia, SIRPIDs appear to be associated with poor outcomeespecially when occurring during hypothermia. ${ }^{2}$

In regards to management, pursuing or not treatment of SIRPIDs is still a matter of debate. The rationale behind this dissensus relies on the fact that there is a lack of evidence on how to care for patients with SIRPIDs ${ }^{6}$; therefore, to improve care, accurate diagnostic and prognostic data should be consistently obtained and, ideally, reported.

In this context, we strongly recommend the routine performance of different noxious stimuli during EEG in comatose patients. Regarding the various possible stimulus types in particular, there has not been a definite agreement on the most efficient method to test nociceptive EEG reactivity. Thus, we highlight the importance of standardizing stimulation parameters in comatose patients, as suggested by Tsetsou et al. ${ }^{7}$ Furthermore, by presenting our patient's EEG, which is fairly typical of SIRPIDs, we intend to remind neurologists of this specific EEG pattern, hoping to encourage collaborative studies that will be able to determine the prognostic and therapeutic implications of SIRPIDs.

\section{Disclosures}

None of the authors have anything to disclose.

\section{REFERENCES}

1. Hirsch LJ, Claassen J, Mayer SA, Emerson RG. Stimulus-induced rhythmic, periodic, or ictal discharges (SIRPIDs): a common EEG phenomenon in the critically ill. Epilepsia. 2004;45:109-23.

2. Alvarez V, Oddo M, Rossetti AO. Stimulus-induced rhythmic, periodic or ictal discharges (SIRPIDs) in comatose survivors of cardiac arrest: characteristics and prognostic value. Clin Neurophysiol. 2013;124:204-8.

3. Lin JH, Chen C, Shyu HY, Kwan SY, Yiu CH. Stimulus-induced rhythmic, periodic, or ictal discharges - a case report. Clin Neurol Neurosurg. 2012;114:1283-6.

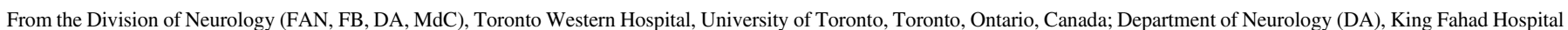
of University, University of Dammam, Saudi Arabia.

Received February 8, 2016. Final Revisions Submitted April 6, 2016. Date of Acceptance May 27, 2016.

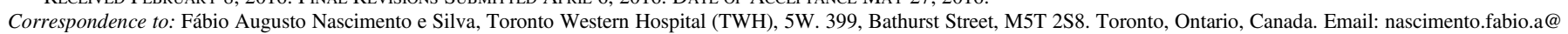
gmail.com 


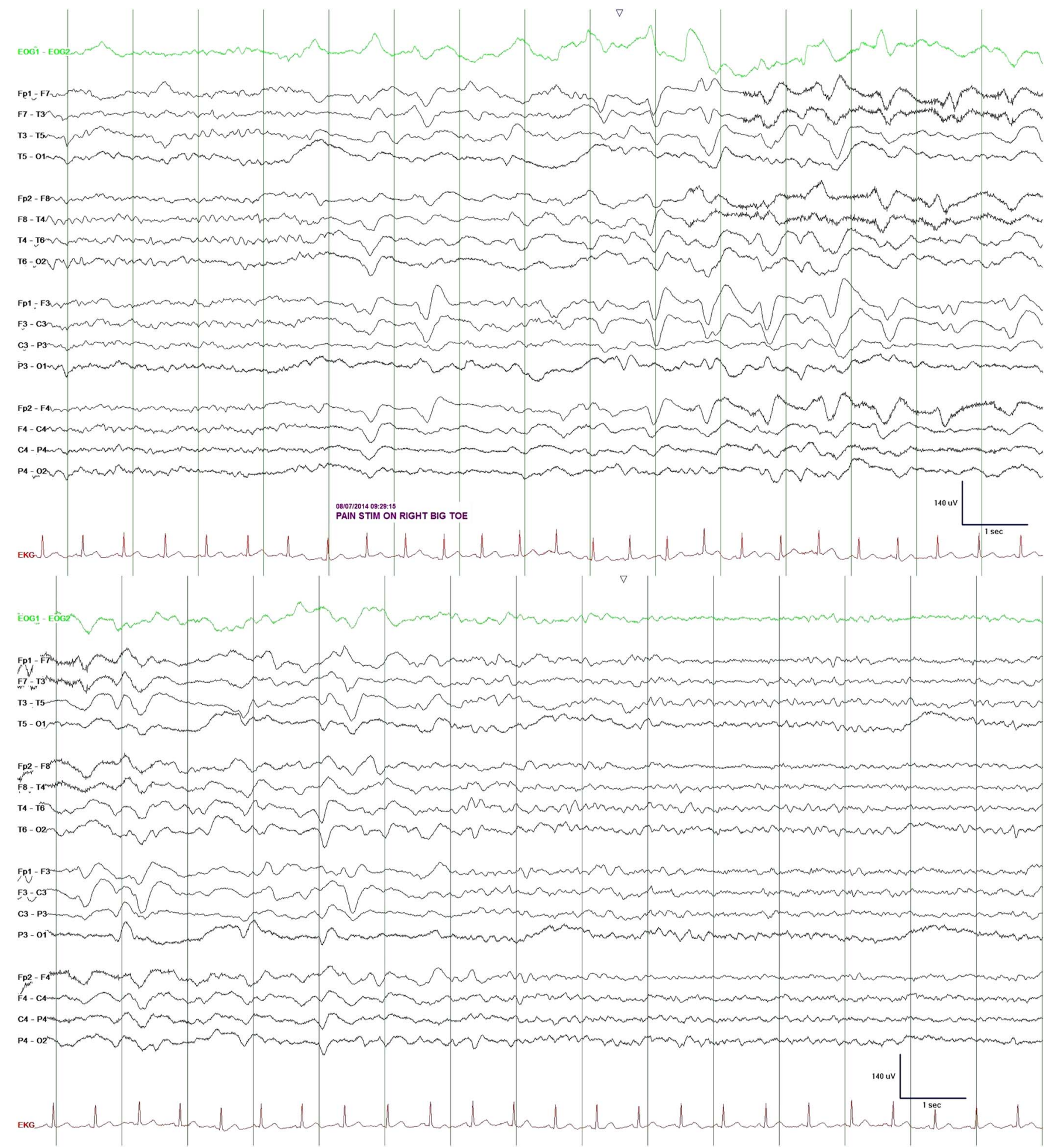

Figure 1: EEG. The patient was comatose throughout the recording. During periods of quiescence, the background activity was $7 \mathrm{~Hz}$ low-amplitude rhythm, mixed with theta and delta frequency waveforms of low amplitude, 1 to $5 \mathrm{~Hz}$, and fairly symmetrical. When the patient was stimulated by applying pain to the extremities, the brain rhythms changed and a pattern of SIRPIDs was noted. The figure shows one of the most typical spells, in which the SIRPIDs pattern was triggered by external painful stimulus to the right hallux. The same pattern was consistently seen in response to different stimuli-auditory as well as painful stimuli-to different sites in the extremities. 


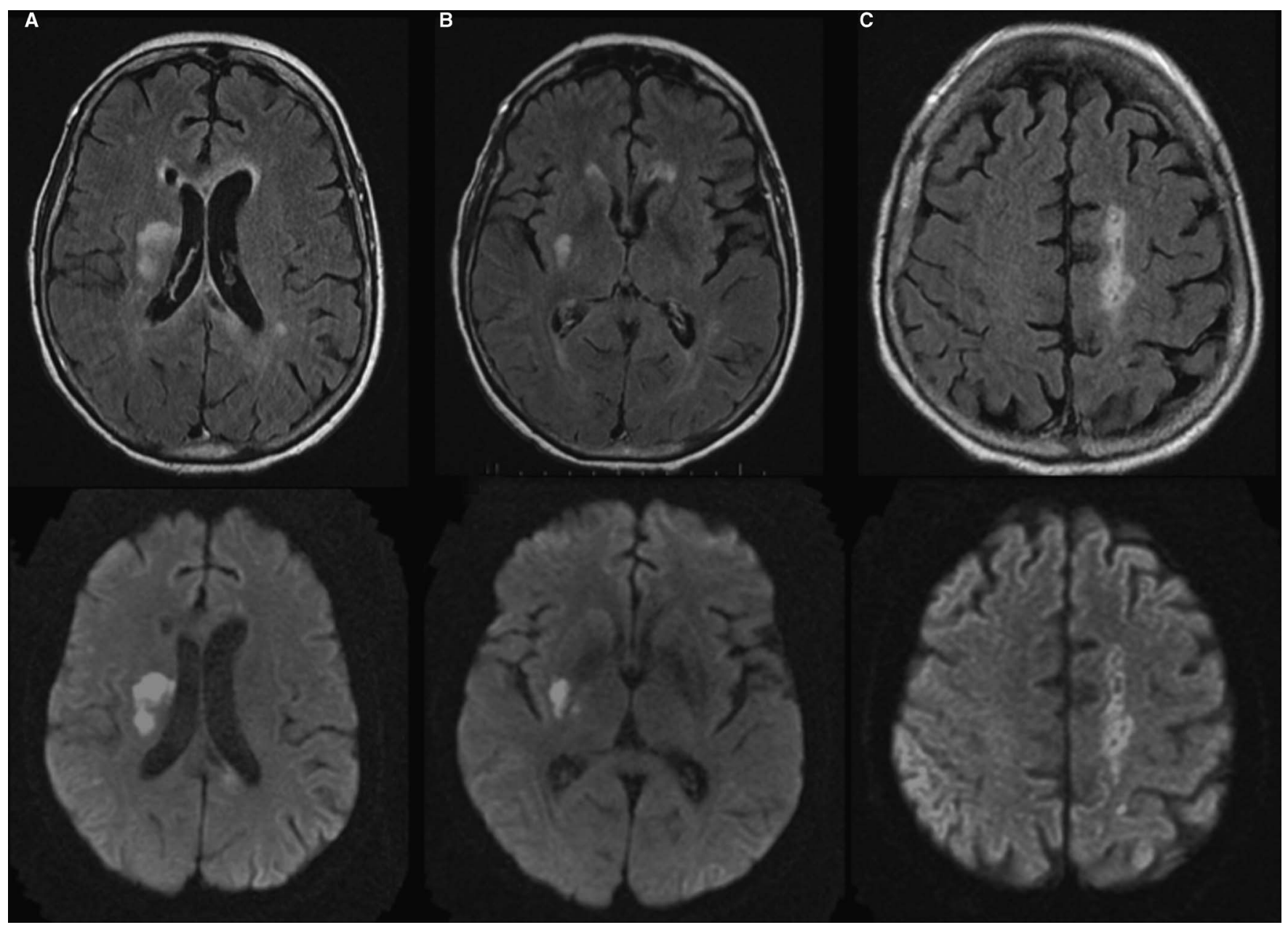

Figure 2: Brain magnetic resonance imaging scan. Axial T2-fluid attenuation inversion recovery weighted (top images) and diffusion-weighted (bottom images). Multiple areas of diffusion restriction with interval evolution involving the right periventricular white matter (A) and extending into the posterior right lentiform nucleus $(B)$. Tiny foci of diffusion restriction were also noted more anteriorly in the right putamen, right frontal operculum, and left parietal lobe (not shown). A previous infarct in the left centrum semiovale has now become associated with encephalomalacia in this area $(C)$.

4. Rossetti AO, Dunand M. Creutzfeldt-Jakob disease: evolution from nonconvulsive status epilepticus, through SIRPIDs, to generalized periodic discharges. Clin Neurophysiol. 2007;118:2533-6.

5. Andraus ME, Andraus CF, Alves-Leon SV. Periodic EEG patterns: importance of their recognition and clinical significance. Arq Neuropsiquiatr. 2012;70:145-51.
6. Van Straten AF, Fesler JR, Hakimi R, Sheng T, Thompson DM, Hakimi AS. SIRPIDs: prevalence and outcome in critically ill patients. J Clin Neurophysiol. 2014;31:418-21.

7. Tsetsou S, Novy J, Oddo M, Rossetti AO. EEG reactivity to pain in comatose patients: Importance of the stimulus type. Resuscitation. 2015;97:34-7. 